Canad. Math. Bull. Vol. 19 (4), 1976

\title{
A MAP OF A POLYHEDRON ONTO A DISK
}

\author{
RICHARD F. E. STRUBE
}

A map $f: X \rightarrow Y$ is said to be universal if for every map $g: X \rightarrow Y$ there exists an $x \in X$ such that $f(x)=g(x)$. In [2] W. Holsztyński observed that if $B$ is a Boltyanskii continuum (see [1]), then there exists a universal map $f: B \rightarrow I^{2}$ such that the product map $f \times f: B \times B \rightarrow I^{2} \times I^{2}$ is not universal. Using this he showed that $B$ can be replaced by a two-dimensional polyhedron. He did not, however, give a concrete example. We exhibit explicitly a two-dimensional polyhedron $K$ and a universal map $f: K \rightarrow I^{2}$ such that $f \times f: K \times K \rightarrow I^{2} \times I^{2}$ is not universal.

Consider the annulus $S^{1} \rtimes I$, with boundary consisting of the circles $S^{1} \times\{0\}$ and $S^{1} \times\{1\}$. Identify every four points of the circle $S^{1} \times\{0\}$ which divide it into four equal arcs, and identify every two points of the circle $S^{1} \times\{1\}$ which divide it into two equal arcs. Let $K$ denote the polyhedron obtained from $S^{1} \times I$ with these identifications. ( $K$ is called a "leaf of degree one" in [1]).

Define a map $f: K \rightarrow I^{2}$ as follows: $f$ maps the image of $S^{1} \times\{0\}$ in $K$ to the centre of $I^{2}, f$ maps the image of $S^{1} \times\{1\}$ in $K$ homeomorphically to the boundary $\dot{I}^{2}$ of $I^{2}$, and $f$ maps the image of a radial line segment from $S^{1} \times\{0\}$ to $S^{1} \times\{1\}$ in $K$ to a radial line segment from the centre to the boundary of $I^{2}$.

Proposition 1. The map $f: K \rightarrow I^{2}$ is a universal map.

Proposition 2. The map $f \times f: K \times K \rightarrow I^{2} \times I^{2}$ is not a universal map.

Lemma 1. A map $f: X \rightarrow I^{n}$ is not universal if and only if there is an extension $F$ of the map $f \mid f^{-1}\left(\dot{I}^{n}\right): f^{-1}\left(\dot{I}^{n}\right) \rightarrow \dot{I}^{n}$ to all of $X$.

Proof. If $f$ is not a universal map then there exists a map $g: X \rightarrow I^{n}$ such that $f(x) \neq g(x)$ for all $x \in X$. Construct a directed line segment from $g(x)$ through $f(x)$, intersecting $\dot{I}^{n}$ at $F(x)$. Then $F$ is the desired extension. If $F$ is such an extension, let $h: \dot{I}^{n} \rightarrow \dot{I}^{n}$ be the antipodal map. Then $(h \circ F)(x) \neq f(x)$ for all $x \in X$, and thus $f$ is not universal.

Proof of Proposition 1. Let $A=f^{-1}\left(\dot{I}^{2}\right)$. By Lemma 1 it suffices to show that we cannot extend $f \mid A: A \rightarrow \dot{I}^{2}$ to a map $F: K \rightarrow \dot{I}^{2}$. For if such an extension

Received by the editors October 8, 1975 . 
existed, we would have a commutative homology triangle

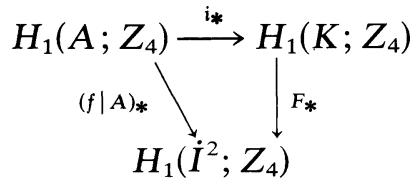

Using the cell structure of $K$ pictured in the proof of the following Lemma 3, $i_{*}\left[2 e_{1}^{1}\right]=i_{*}\left[2 e_{1}^{1}+4 e_{2}^{1}\right]=i_{*}\left[\partial e_{1}^{2}\right]=0$. Since $(f \mid A)_{*}\left[e_{1}^{1}\right]=\left[f_{1}^{1}\right]$, this would imply that $2\left[f_{1}^{1}\right]=0$, a contradiction.

Proposition 2 is an immediate consequence of the following two lemmas. Let $C=(f \times f)^{-1}\left(\dot{I}^{4}\right)$, and let $s^{*}$ be a generator of $H^{3}\left(S^{3}\right)$ (we use integral coefficients).

Lemma 2. $\delta(f \times f \mid C)^{*}\left(s^{*}\right)=0$ in $H^{4}(K \times K, C)$ if and only if $f \times f$ is not universal.

Proof. By the Hopf extension theorem (see Spanier, [4]), $\delta(f \times f \mid C)^{*}\left(s^{*}\right)=$ 0 if and only if the map $f \times f \mid C$ can be extended over $K \times K$. The lemma then follows from Lemma 1.

Lemma 3. $\delta(f \times f \mid C)^{*}\left(s^{*}\right)=0$ in $H^{4}(K \times K, C)$.

Proof. Consider the diagram

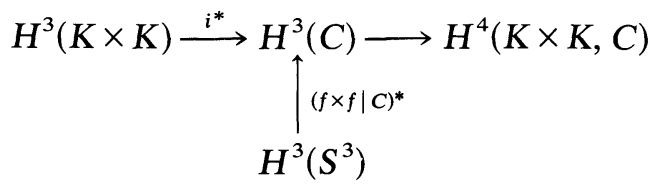

Since $\operatorname{Ker} \delta=\operatorname{Im} i^{*}$, it suffices to show that $(f \times f \mid C)^{*}\left(s^{*}\right)$ is in $\operatorname{Im} i^{*}$. Give $K$ and $I^{2}$ the cell structure indicated below $(K$ is a regular cell complex with identifications and the arrows give the orientations of the cells).

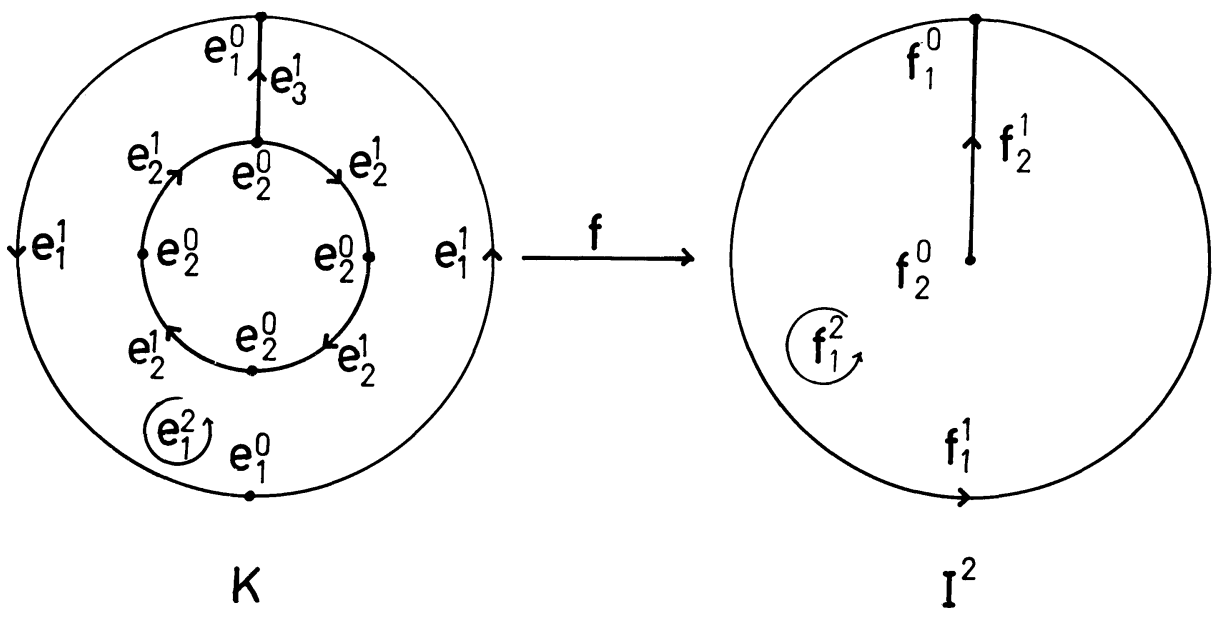


Then $f_{*}: C_{*}(K) \rightarrow C_{*}\left(I^{2}\right)$ maps $e_{1}^{0}$ to $f_{1}^{0}, e_{2}^{0}$ to $f_{2}^{0}, e_{1}^{1}$ to $f_{1}^{1}, e_{2}^{1}$ to $0, e_{3}^{1}$ to $f_{2}^{1}$, and $e_{1}^{2}$ to $2 f_{1}^{2}$. Choose ordered bases of oriented cells: $\left\{\alpha_{1}, \alpha_{2}\right\}$ for $C_{3}(C)$, $\left\{\beta_{1}, \ldots, \beta_{7}\right\}$ for $C_{2}(C),\left\{\gamma_{1}\right\}$ for $C_{4}(K \times K),\left\{\delta_{1}, \ldots, \delta_{6}\right\}$ for $C_{3}(K \times K),\left\{\varepsilon_{1}, \varepsilon_{2}\right\}$ for $C_{3}\left(S^{3}\right)$, and $\left\{\phi_{1}, \ldots, \phi_{5}\right\}$ for $C_{2}\left(S^{3}\right)$. For appropriate choices of these bases we have

$$
\partial \alpha_{1}=2 \beta_{1} \quad \partial \alpha_{2}=4 \beta_{2} \quad \partial \gamma_{1}=2 \delta_{1} \quad \partial \varepsilon_{1}=\phi_{1} \quad \partial \varepsilon_{2}=-\phi_{1}
$$

and

$$
\begin{array}{ll}
i_{*}\left(\alpha_{1}\right)=\delta_{1}-\delta_{4}-2 \delta_{5}-2 \delta_{6} & i_{*}\left(\alpha_{2}\right)=-\delta_{1}+2 \delta_{5}+2 \delta_{6} \\
(f \times f)_{*}\left(\alpha_{1}\right)=2 \varepsilon_{1} & (f \times f)_{*}\left(\alpha_{2}\right)=-2\left(\varepsilon_{1}+\varepsilon_{2}\right)
\end{array}
$$

Let $\left(k_{1}, \ldots, k_{n}\right)$ in $\operatorname{Hom}\left(Z^{n}, Z\right)$ denote the homomorphism which multiplies the $i$ th component by $k_{i}$. Then a generator $s^{*}$ of $H^{3}\left(S^{3}\right)$ is given by $[(1,0)]$, and hence $(f \times f \mid C)^{*}\left(s^{*}\right)=[(2,-2)]$. This element is not zero in $H^{3}(C)$ : for $\left(k_{1}, \ldots, k_{7}\right)=\left(2 k_{1}, 4 k_{2}\right)=(2,-2)$ for any choice of $\left(k_{1}, \ldots, k_{7}\right)$ in $\operatorname{Hom}\left(C_{2}(C), Z\right)$. However, if $\left(k_{1}, \ldots, k_{6}\right) \in \operatorname{Hom}\left(C_{3}(K \times K), Z\right)$, then $i^{*}\left(k_{1}, \ldots, k_{6}\right)=\left(k_{1}-k_{4}-2 k_{5}-2 k_{6},-k_{1}+2 k_{5}+2 k_{6}\right)$. Since $\delta\left(k_{1}, \ldots, k_{6}\right)\left(\gamma_{1}\right)=$ $2 k_{1},\left(k_{1}, \ldots, k_{6}\right)$ represents a cocycle in $\operatorname{Hom}\left(C_{3}(K \times K), Z\right)$ if and only if $k_{1}=0$. Hence $i^{*}\left[\left(k_{1}, \ldots, k_{6}\right)\right]=[(2,-2)]$ if and only if $k_{1}=0, k_{4}=0$, and $k_{5}+k_{6}=-1$. In particular, $i^{*}[(0,0,0,0,0,-1)]=[(2,-2)]=(f \times f \mid C)^{*}\left(s^{*}\right)$ and hence $(f \times f \mid C)^{*}\left(s^{*}\right) \in \operatorname{Im} i^{*}$.

Since $K$ can be imbedded in $R^{4}$, taking the double of a closed regular neighbourhood of $K$ in $R^{4}$ leads to a concrete example of a closed 4-manifold $M^{4}$ and a universal map $g: M^{4} \rightarrow I^{2}$ such that $g \times g: M^{4} \times M^{4} \rightarrow I^{2} \times I^{2}$ is not a universal map (compare [3]).

\section{REFERENCES}

1. V. Boltyanskiŭ, An example of a two-dimensional compactum whose topological square is three-dimensional, Doklady Akad. Nauk SSSR (N.S.) 67 (1949), 597-599. [Amer. Math. Soc. Transl.]

2. W. Holsztyński, Universal mappings and fixed point theorems, Bull. Acad. Polon: Sci. Sér. Sci. Math. Astronom. Phys. 15 (1967), 433-438.

3. W. Holsztyński, On the product and composition of universal mappings of manifolds into cubes, Proc. Amer. Math. Soc. 58 (1976), 311-314.

4. E. Spanier, Algebraic Topology, McGraw-Hill, 1966.

Department of Mathematics

UNIVERSITY OF WESTERN ONTARIO

LONDON, ONTARIO, N6A 3K7

CANADA 